Revue

Revue de l'histoire des religions

de Ihistoire des religions

Jacques RIVIÈRE, La Théodicée de Fénelon. Ses éléments quiétistes. Suivi de Fénelon 1908. Jacques Rivière philosophe, par François TRÉMOLIÈRES

Paris, Le Félin, 2015

Jacques Le Brun

\title{
CpenEdition
}

Journals

Édition électronique

URL : http://journals.openedition.org/rhr/8811

DOI : $10.4000 /$ rhr.8811

ISSN : 2105-2573

Éditeur

Armand Colin

Édition imprimée

Date de publication : 1 septembre 2017

Pagination : $576-579$

ISBN : 978-2-200-93127-8

ISSN : 0035-1423

Référence électronique

Jacques Le Brun, « Jacques Rivière, La Théodicée de Fénelon. Ses éléments quiétistes. Suivi de

Fénelon 1908. Jacques Rivière philosophe, par François TrémoLières », Revue de l'histoire des religions [En ligne], 3 | 2017, mis en ligne le 01 septembre 2017, consulté le 06 janvier 2021. URL : http:// journals.openedition.org/rhr/8811 ; DOI : https://doi.org/10.4000/rhr.8811

Ce document a été généré automatiquement le 6 janvier 2021

Tous droits réservés 


\section{Jacques RIVIÈRE, La Théodicée de Fénelon. Ses éléments quiétistes. Suivi de Fénelon 1908. Jacques Rivière philosophe, par François TRÉMOLIÈRES}

Paris, Le Félin, 2015

Jacques Le Brun

\section{RÉFÉRENCE}

Jacques RIvière, La Théodicée de Fénelon. Ses éléments quiétistes. Suivi de Fénelon 1908.

Jacques Rivière philosophe, par François TRÉMOLIÈrEs, Paris, Le Félin, 2015, 23 cm, 480 p., $25 €$, ISBN 978-2-86645-830-0.

À partir de décembre 1908, Jacques Rivière, le futur directeur de la N.R.F., publiait dans les Annales de Philosophie chrétienne en plusieurs livraisons, sous le titre de «La Théodicée de Fénelon. Ses éléments quiétistes", le texte d'un Diplôme d'études supérieures soutenu quelques mois auparavant, le 20 juin, en Sorbonne. C'est le texte publié dans ce périodique qui, à défaut du manuscrit perdu, est ici donné par François Trémolières et sert à ce dernier de point de départ pour un ensemble imposant de recherches pour ainsi dire concentriques sur l'auteur, ses amis et correspondants, la philosophie, les sciences religieuses aux XIX ${ }^{e}$ et $\mathrm{Xx}^{\mathrm{e}}$ siècles et les études féneloniennes. Travail imposant, car, à partir de cette référence qu'est le mémoire de Rivière, il couvre une longue période et tout un ensemble de disciplines et de travaux inexplorés jusqu'à son enquête. Cercles concentriques, disions-nous, car chaque recherche en appelle une autre, aboutissant, par la mise en rapport des unes avec les autres et de chacune avec Rivière, à un vaste tableau de la vie intellectuelle, philosophique, littéraire et religieuse en France jusqu'à la seconde moitié du xx siècle. 
2 Le premier cercle, c'est le texte même publié par Rivière en 1908. Le jeune candidat au D.E.S. y présente d'abord, selon la chronologie des œuvres, la théodicée de Fénelon selon la Réfutation de Malebranche, puis selon le Traité de l'existence de Dieu et les autres textes philosophiques. À l'exposé et au commentaire de ces textes Rivière joint la présentation de ceux qu'il appelle "quiétistes", orientant avec ce terme, qui au $\mathrm{XVII}^{\mathrm{e}}$ siècle avait une valeur polémique, son interprétation dans le sens d'une hétérodoxie de Fénelon, la passivité, l'union à Dieu, le pur amour. Cela permet à Rivière de conclure que la théodicée de Fénelon est encore plus "quiétiste» que le "quiétisme" lui-même, conclusion qui laisse penser qu'une tendance panthéiste, refoulée ou voilée, est au centre de la pensée de Fénelon, mais aussi qui soutient que l'origine de tout le « système » de Fénelon c'est l'amour pur, le primat de l'amour sur la vérité, conclusion qui lie philosophie, théologie et spiritualité.

Le lecteur du mémoire est alors en mesure de suivre le véritable livre qu'en plus de 300 pages et en une multiplicité de points de vue François Trémolières nous donne à lire. Il le fait en se centrant d'abord sur la genèse du mémoire, les circonstances de sa rédaction et de sa publication. Pour faire diriger et reconnaitre son travail Rivière s'était d'abord adressé à Victor Delbos qui lui conseilla de se tourner vers Alfred Rébelliau, choix qui se révèlera décisif : le travail de Rébelliau, auteur d'un D.E.S. sur Malebranche et d'une thèse qui a fait date sur Bossuet (elle sera rééditée revue et augmentée en 1909), était orienté vers la littérature, l'histoire et cette discipline au statut encore incertain mais non confessionnel qu'étaient les sciences religieuses. Ainsi François Trémolières s'attachera à exposer l'état de la philosophie en France à la fin du XIX siècle et l'émergence d'une science des religions sur l'échec de la "science catholique » que l'Église catholique avait tenté de promouvoir au milieu du siècle. Tel était le cadre disciplinaire dans lequel se situait le mémoire de Rivière. Mais plus que le genre de la "dissertation » universitaire c'est la littérature qui déjà attirait Rivière : Barrès, et surtout Claudel et Gide (les correspondances avec eux permettent de jalonner l'évolution) seront en quelque sorte les figures tutélaires qui l'accompagneront dans le chemin depuis la philosophie et la pensée religieuse vers une vie toute entière consacrée à la littérature.

4 Avant de parcourir avec une remarquable érudition et à partir de sources de première main cet itinéraire, François Trémolières s'attache sous le titre de "Critique » à faire l'exégèse du mémoire tel que publié dans les Annales de Philosophie chrétienne. Comme le titre l'indique, la thèse de Rivière tournera autour du rapport entre deux orientations de l'œuvre et de la pensée de Fénelon, la théodicée et les « éléments quiétistes ", c'està-dire les écrits qui en leur temps furent suspectés de "quiétisme». Sur la théodicée Rivière adopte un point de vue génétique, l'idée d'un développement chronologique depuis la Réfutation de Malebranche jusqu'à la Démonstration, mais c'est pour mieux souligner une identité entre la théodicée, c'est-à-dire les aspects philosophiques, et les textes mystiques ou portant sur la mystique. La question centrale qui apparait dès les premières pages du mémoire c'est celle du rapport entre Dieu et le monde, immanence (et confusion) ou transcendance (et liberté) : ainsi dès la page 46 l'affirmation, voilée de réticences (" comment hésiter à dire... »), selon laquelle la théodicée de Fénelon a tous les caractères du panthéisme, depuis la Réfutation jusqu'aux écrits ultérieurs, les thèses réfutées dans la première devenant centrales dans les seconds. Mais ici l'exégète souligne avec Rivière qu'un «reste de ses premières conceptions " reste perceptible chez Fénelon. Là un pas est franchi par Rivière dans son interprétation, ouvrant des 
perspectives sur ce qui de l'intérieur peut animer le mémoire: Rivière imagine des motifs psychologiques expliquant chez Fénelon une sorte de vacillement, permanence et retournement pouvant subsister ensemble et il préfère parler de « tendance » plutôt que de " confusion », et dire que ce panthéisme serait « inconscient ».

5 Le «quiétisme » est le deuxième pan de l'ensemble élaboré par Rivière, mais déjà ce terme de «quiétisme » n'est-il pas une interprétation ? C'était l'accusation lancée par les adversaires de Fénelon qui voulaient mettre en lumière chez l'archevêque la résurgence des erreurs de Molinos condamnées en 1687 dans la constitution Coelestis Pastor, accusation que Fénelon et ses défenseurs ont toujours écartée. Quelle que soit la pertinence de ce terme de "quiétisme", c'est bien un ensemble marqué par le panthéisme qui unifie selon Rivière les deux horizons de l'œuvre de Fénelon. Un point sur lesquels il appuie, et cela avec une grande pertinence, son analyse c'est la confrontation entre Fénelon et Malebranche. Le dialogue inauguré avec la Réfutation se poursuivrait souterrainement dans les œuvres philosophiques. Mais ici, reprenant les choses avec des nuances, voire des hésitations perceptibles derrière les juvéniles affirmations, Rivière décèle des convergences inattendues entre cette Réfutation et les œuvres qui semblent contredire cette dernière. L'importance de mettre au jour ces convergences est d'importance primordiale pour Rivière, et cela aussi bien pour la mystique que pour la philosophie : la passivité du mystique répondrait à celle de la nature, l'union des volontés avec Dieu à l'union essentielle. Serait-ce alors aux yeux de Rivière cohabitation de thèses contradictoires? ou visée du point où les contradictions s'ordonnent? C'est en tout cas une façon d'ancrer le pur amour non seulement dans l'expérience du mystique, mais surtout dans l'immanence, façon de marquer la prééminence du philosophique sur le spirituel.

6 L'analyse du mémoire, au-delà des nuances et de la finesse du travail de Rivière, ne laisse pas de doute sur la conviction de son auteur: la pensée de Fénelon est hétérodoxe ou tend à l'hétérodoxie. Devant cette conviction, François Trémolières est conduit à mener une vaste enquête à travers la philosophie et la théologie du XIX siècle, de deux points de vue : du point de vue de l'interprétation de Fénelon, et du point de vue de l'histoire des débats autour du "panthéisme "; tâche immense qu'il mène avec une impressionnante érudition et qui nous fait découvrir des continents encore peu explorés ou des œuvres qui n'ont pas souvent été mises en rapport les unes avec les autres : les œuvres des Villemain, des Bautain, des Maret, jusqu'au milieu intellectuel où se situent les Annales de Philosophie chrétienne. Et en même temps c'est l'histoire de la constitution d'un « Fénelon philosophe » un siècle avant qu'Henri Gouhier ne donne en 1977 sous ce titre un maitre livre.

7 À l'enquête sur la constitution au XIX ${ }^{e}$ siècle d'un Fénelon proche du "panthéisme " et sur le moment intellectuel, philosophique, littéraire et religieux de la publication (autour de 1908), se joindra une enquête parallèle, en quelque sorte un des cercles que nous évoquions, sur la réception du mémoire et de la thèse qu'il promouvait. Mais avant la réception de ces articles, c'est chez Rivière lui-même que la publication de 1908 marque une date décisive : d'un côté c'est la thèse, implicite mais d'autant plus personnelle qu'au-delà de Fénelon c'est le christianisme (le christianisme "théologique ", mais quelles en seraient les limites ?) qui tend au panthéisme; d'un autre côté c'est un tournant, dont la correspondance avec Claudel porte témoignage, tournant vers la littérature : un jalon serait le texte, trop peu connu, rédigé par Rivière en parallèle avec le mémoire, l'Introduction à la métaphysique du rêve; ce texte, prenant 
en compte l'importance d'une autre mystique, celle qu'avait vécue Rimbaud, est un bon témoin du passage par le rêve (par l'inconscient ?) d'un point de vue « théologique » et "philosophique » à un point de vue "littéraire », avec l'interrogation : quel rapport, intérieur au langage et aux affects, au « réel »?

8 La réception des thèses de Rivière se fera aussi dans le champ des études féneloniennes. En 1910-1911 la Revue Fénelon (où pourtant écrivirent Rébelliau, Levesque, Bremond) ne cite nulle part les articles de Rivière de 1908-1909, mais des œuvres majeures laissent percevoir l'écho plus ou moins souterrain d'un dialogue avec eux. Le grand livre de Delacroix de 1908, l'Apologie pour Fénelon de Bremond de 1910, les travaux universitaires du $\mathrm{Xx}^{\mathrm{e}}$ siècle montrent que, même peu citées, les orientations données par Rivière, et sans doute malgré lui, ont permis aux études féneloniennes de gagner en profondeur. Sur toutes ces questions François Trémolières nous donne un livre fondamental, d'une impressionnante érudition, qui marquera à la fois ces études féneloniennes et les recherches sur la philosophie et les sciences religieuses aux XIX ${ }^{\mathrm{e}}$ et $\mathrm{XX}^{\mathrm{e}}$ siècles.

\section{AUTEURS}

\section{JACQUES LE BRUN}

École Pratique des Hautes Études, Paris. 